\title{
Diafragma. Anatomía y evaluación por ecografía
}

\section{Diaphragm. Anatomy and evaluation by ultrasound}

Aloisia P. Hernández-Morales*, Lya E. Pensado-Piedra, Fortunato Juárez-Hernández y

Roberto Sotelo-Robledo

Departamento de Imagenología, Instituto Nacional de Enfermedades Respiratorias (INER), Ciudad de México, México

\section{RESUMEN}

La creciente aceptación del ultrasonido en la evaluación del tórax ha permitido ser más certeros en el diagnóstico de los pacientes con patología torácica. Así, el ultrasonido en la evaluación del diafragma, como principal músculo respiratorio, facilita un diagnóstico fácil y reproducible, con una curva de aprendizaje corta. Esta revisión proporciona las características de imagen por ultrasonido del diafragma en cuanto a su descripción anatómica y funcional.

Palabras clave: Diafragma. Contracción. Excursión. Grosor diafragmático.

\section{ABSTRACT}

The growing acceptance of ultrasound in the evaluation of the thorax has allowed us to be more accurate in the diagnosis of patients with thoracic pathology; thus, ultrasound in the evaluation of the diaphragm, as the main respiratory muscle, facilitates an easy and reproducible diagnosis, with a short learning curve. So this review provides the ultrasound imaging characteristics of the diaphragm in terms of its anatomical and functional description.

Keywords: Diaphragm. Contraction. Excursion. Diaphragmatic thickness. 


\section{INTRODUCCIÓN}

La evaluación ecográfica del diafragma ha tenido una creciente aceptación, ya que permite el reconocimiento rápido, fácil y certero de su morfología y función, tanto en pacientes en situación crítica como aquellos hemodinámicamente estables.

Existen numerosas enfermedades que pueden lesionar el diafragma, que van desde las enfermedades que afectan el sistema nervioso central y periférico hasta aquellas que causan propiamente disfunción muscular, como las distrofias musculares y las enfermedades de la unión neuromuscular. Este espectro de enfermedades condiciona disfunción diafragmática, que se define como una pérdida de la fuerza muscular que origina una reducción en la capacidad inspiratoria, limitando la resistencia muscular respiratoria.

Actualmente, una de las aplicaciones más relevantes de la evaluación ecográfica del diafragma es la predicción de disfunción diafragmática inducida por el ventilador. La lesión diafragmática inducida por el ventilador puede estar presente en cerca del 80\% de los pacientes en unidades de terapia intensiva, y ocurre tan temprano como en las primeras 24-36 horas después de iniciada la ventilación mecánica.

En esta revisión mostramos el uso del ultrasonido en el diagnóstico de parálisis diafragmática y disfunción diafragmática, y su uso potencial en pacientes en la unidad de cuidados intensivos.

\section{MÉTODO}

Se realizó una amplia revisión bibliográfica en la base de datos PubMed, donde intencionadamente se indagó sobre las primeras descripciones ecográficas, usando las siguientes palabras clave: disfunción diafragmática, ultrasonido diafragmático y sonografía diafragmática; incluyendo estudios originales, editoriales y revisiones bibliográficas. En la mayoría de los estudios, la función del diafragma fue estimada por observación directa de la excursión diafragmática, mientras en pequeñas series se evaluó a través de las estructuras anatómicas vecinas, como el hígado y el bazo (técnica indirecta); así mismo, se evaluó el grosor diafragmático.

\section{REVISIÓN}

\section{Anatomía}

El diafragma es una estructura musculotendinosa con forma de domo, que separa el tórax del abdomen. Es el principal musculo inspiratorio y actúa como barrera para la transmisión de la presión abdominal y torácica.

El diafragma consta de un componente muscular y un tendón central. El componente muscular puede subdividirse en dos partes: el diafragma costal y el diafragma crural. El diafragma costal se origina anterior al esternón, lateralmente a lo largo de la superficie interna de las seis costillas inferiores y anteromedialmente a lo largo de los cartílagos costales. El diafragma crural se origina posteriormente a lo largo de los tres cuerpos vertebrales lumbares superiores y da origen a los ligamentos arcuatos medial y lateral. 
El componente muscular del diafragma se inserta en el tendón central fibroso, el cual tiene una delgada, pero fuerte, aponeurosis. El tendón central del diafragma varía en tamaño según los individuos: puede comprender menos del 10\% del diafragma a más del 35\% del en algunos individuos, y generalmente tiene forma de V. Además, tiene varios orificios, llamados hiatos, que permiten la comunicación entre las cavidades abdominal y torácica.

El nervio frénico inerva el diafragma. Principalmente es un nervio motor que se origina de C3, C4 y C5, con la mayoría de las motoneuronas originadas en $\mathrm{C} 4$. Ambos nervios frénicos, derecho e izquierdo, se dividen en tres a cinco ramos que inervan las porciones anterolateral, posteromedial, esternal y crural del diafragma. Los ramos entonces cursan dentro del músculo diafragmático y corren en un plano medial entre la pleura y las capas de peritoneo ${ }^{1}$.

Fisiológicamente, cuando se contrae, se mueve en sentido caudal, incrementando la presión intraabdominal y disminuyendo la presión intrapleural. Esta reducción en la presión pleural resulta en una disminución en la presión intraalveolar. Una vez la presión intraalveolar se hace subatmosférica, el aire entra al alveolo ${ }^{1}$. Entonces, el diafragma se relaja y asciende durante la espiración, permitiendo la salida del aire. También ayuda a incrementar la presión abdominal para la defecación, la micción y el llanto, y trabaja en conjunto con el esfínter esofágico al ejercer presión en el hiato esofágico y prevenir el reflujo ${ }^{2}$.

Normalmente, durante el sueño de movimientos rápidos del ojo, el diafragma es el único musculo inspiratorio que funciona y los músculos inspiratorios accesorios permanecen inactivos. En consecuencia, los individuos con debilidad o parálisis diafragmática pueden presentarse con síntomas de hipoventilación, tales como despertares nocturnos frecuentes, nocturia, pesadillas vividas, sudoración nocturna, hipersomnolencia durante el día, depresión y cefalea matutina ${ }^{3}$.

En general, inicialmente debe tomarse una radiografía de tórax posteroanterior ante la sospecha de patología diafragmática, con el objetivo de:

- Observar patología diafragmática, frecuentemente incidental

- Dar por hecho que el diafragma no es directamente visible.

- Tratar de decidir si la anormalidad es, en efecto, localizada en el diafragma, o secundaria a otra enfermedad adyacente al diafragma.

Debido a la variable presentación del diafragma, muchos cambios vistos en una radiografía de tórax no están relacionados con una patología, sea o no importante, y entonces las decisiones deben tomarse sobre la importancia del hallazgo y si es necesario realizar una modalidad de imagen adicional, como una evaluación ecográfica.

En la tabla 1 se muestran las indicaciones para la evaluación ecográfica del diafragma.

\section{Evaluación ecográfica}

Por ecografía, el diafragma puede verse con una técnica directa que incluye la evaluación de su grosor, excursión e integridad, o con una técnica indirecta en la que se usa otra 


\begin{tabular}{|c|c|}
\hline \multirow[t]{3}{*}{ Indicación total } & $\begin{array}{l}\text { Diagnóstico y monitoreo de parálisis } \\
\text { diafragmática }\end{array}$ \\
\hline & $\begin{array}{l}\text { Elevación diafragmática en la radiografía de } \\
\text { tórax }\end{array}$ \\
\hline & Disnea de causa desconocida \\
\hline \multirow{4}{*}{$\begin{array}{l}\text { Indicación } \\
\text { médica }\end{array}$} & Paro con falla respiratoria \\
\hline & Trastorno neuromuscular \\
\hline & Guía para aguja de electromiografía \\
\hline & Evaluación de enfermedad crónica (EPOC) \\
\hline \multirow{2}{*}{$\begin{array}{l}\text { Indicación } \\
\text { quirúrgica }\end{array}$} & Rotura diafragmática traumática \\
\hline & Detección de complicación posoperatoria \\
\hline \multirow[t]{4}{*}{$\mathrm{UCl}$} & Dificultad para el destete \\
\hline & Estimación del trabajo ventilatorio \\
\hline & $\begin{array}{l}\text { Estimación de disfunción diafragmática } \\
\text { inducida por el ventilador }\end{array}$ \\
\hline & Valoración del soporte ventilatorio \\
\hline
\end{tabular}

EPOC: enfermedad pulmonar obstructiva crónica; UCl: unidad de cuidados intensivos.

estructura anatómica de referencia para el desplazamiento craneocaudal, como la rama intrahepática izquierda, la excursión del hilio esplénico, el hígado, el páncreas y los riñones.

Para su completa evaluación, tanto morfológica como funcional, el diafragma debe ser visualizado con dos abordajes: el domo y el músculo costal diafragmático (Fig. 1).

El domo diafragmático se observa fácilmente en el área subcostal, entre la línea media clavicular, usando el hígado o el bazo como ventana acústica, con un transductor de baja frecuencia de 2-5 MHz, e inclinando el transductor en sentido cefálico y medial. El diafragma se identifica como una línea hiperecoica, convexa y continua (Fig. 2A). Con este abordaje se evalúan su integridad,

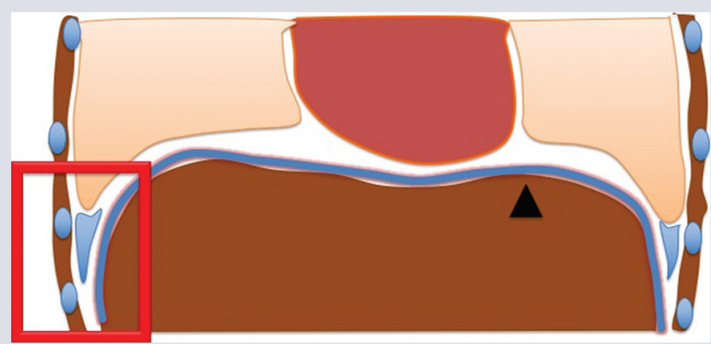

Figura 1. Esquema que enfatiza el abordaje ecográfico del diafragma. La punta de flecha señala el domo diafragmático y en el recuadro rojo se indica la zona de aposición diafragmática.

ecogenicidad y movimiento cefalocaudal en modo $\mathrm{M}$.

El movimiento diafragmático inspiratorio es caudal, y por tanto el diafragma se mueve hacia el transductor. En espiración, el movimiento es cefálico, por lo que se aleja del transductor. En modo $\mathrm{M}$ se usa para la evaluación de la excursión diafragmática (desplazamiento, cm). Con este desplazamiento cefalocaudal diafragmático y su curva sinusoidal se evalúan la velocidad de contracción (pendiente, $\mathrm{cm} / \mathrm{s}$ ), el tiempo inspiratorio (Tinsp, s) y la duración del ciclo (Ttot, s) (Fig. 2B) ${ }^{4}$.

En los sujetos sanos, la medida de la excursión en respiración espontánea y en decúbito supino es de $16 \mathrm{~mm}$ en los hombres y $18 \mathrm{~mm}$ en las mujeres ${ }^{5}$. Esta medida varía de acuerdo con la técnica respiratoria deseada en el paciente para la completa evaluación de la patología diafragmática. Con la técnica del olfateo, varía de 26 a $29 \mathrm{~mm}$, y en respiración forzada es de $58 \mathrm{~mm}$ en las mujeres y $70 \mathrm{~mm}$ en los hombres ${ }^{5}$. Sin embargo, estudios previos han reportado valores promedio de excursión de $42 \pm 16$ a $79 \pm 13 \mathrm{~mm}$ en respiración forzada y de $11 \pm 4 \mathrm{~mm}$ a $21 \pm 6 \mathrm{~mm}$ en respiración espontánea 6 . 


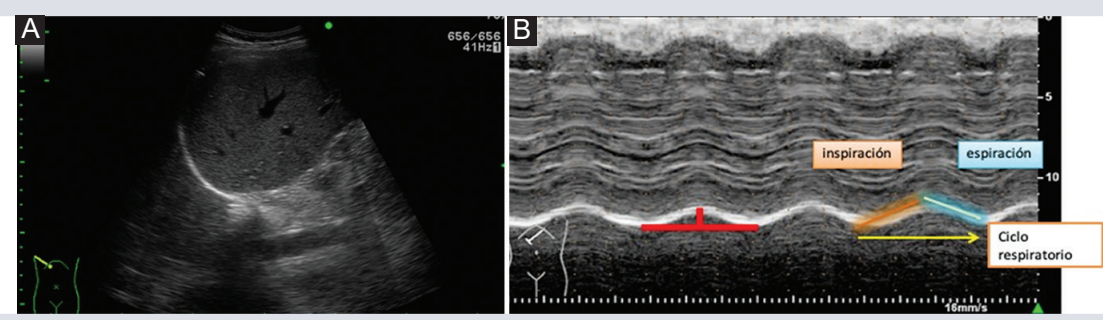

Figura 2. A: imagen ecográfica del domo diafragmático. En este abordaje se evalúan su continuidad, ecogenicidad, integridad y movimiento cefalocaudal. B: modo M. En el trazo del movimiento, la altura máxima es excursión, y el ciclo es la suma de inspiración (naranja) y espiración (azul).

El movimiento del domo diafragmático no es uniforme. Típicamente hay un mayor movimiento caudal de la región medial del domo y de la parte posterior que de su margen anterior. El movimiento estimado de la parte posterior del diafragma es un $40 \%$ mayor que el de su parte anterior ${ }^{5}$.

Hay una excursión similar de los dos hemidafragmas, aunque el movimiento del hemidafragma izquierdo puede ser ligeramente mayor que el del derecho.

El domo diafragmático derecho es $1.9 \mathrm{~cm}$ más cefálico que el diafragma izquierdo al final de la espiración y $1.3 \mathrm{~cm}$ al final de la inspiración durante la respiración espontánea. En pacientes ventilados mecánicamente, anestesiados y con parálisis, la diferencia del lado derecho y del lado izquierdo es de 1.7 y $1.6 \mathrm{~cm}$ al final de la espiración y al final de la inspiración, respectivamente. En supino, en pacientes ventilados, anestesiados o paralizados, las regiones dependientes muestran menor movimiento diafragmático ${ }^{5}$.

La representación en modo $\mathrm{M}$ de una maniobra en espiración forzada (después de una inspiración máxima) se caracteriza por una pendiente inicial seguida por una meseta en espiración máxima. Esta curva es similar a la obtenida en la medición de tiempo/volumen en la espirometría. El análisis de esta curva espirométrica permite medir el volumen espiratorio en el primer segundo $\left(\mathrm{VEF}_{1}\right)$ y la capacidad vital (VC), y calcular la relación $\mathrm{FEV}_{1} / \mathrm{VC}^{6}$.

Observaciones preliminares de la excursión diafragmática en modo $\mathrm{M}$ durante la espiración en voluntarios sanos y pacientes afectados por enfermedad de la vía aérea obstructiva indican que la caída inicial de la curva en espiración es más profunda en los sujetos sanos que en aquellos con enfermedad obstructiva de la vía aérea, lo que sugiere que la excursión puede ser más tardía en los pacientes con obstrucción de la vía aérea y atrapamiento aéreo ${ }^{6}$.

El segundo abordaje para la visualización del diafragma costal es la zona de aposición diafragmática: entre el octavo y el décimo espacios intercostales, línea axilar anterior o medial, $0.5-2 \mathrm{~cm}$ por debajo del seno costofrénico (Fig. 3). Con este abordaje se evalúa el grosor; lo normal en esta zona es $1.7 \pm 0.2 \mathrm{~cm}$ en respiración relajada, y se incrementa a $4.5 \pm$ $0.9 \mathrm{~mm}$ cuando se realiza una respiración a capacidad pulmonar total ${ }^{5}$. 


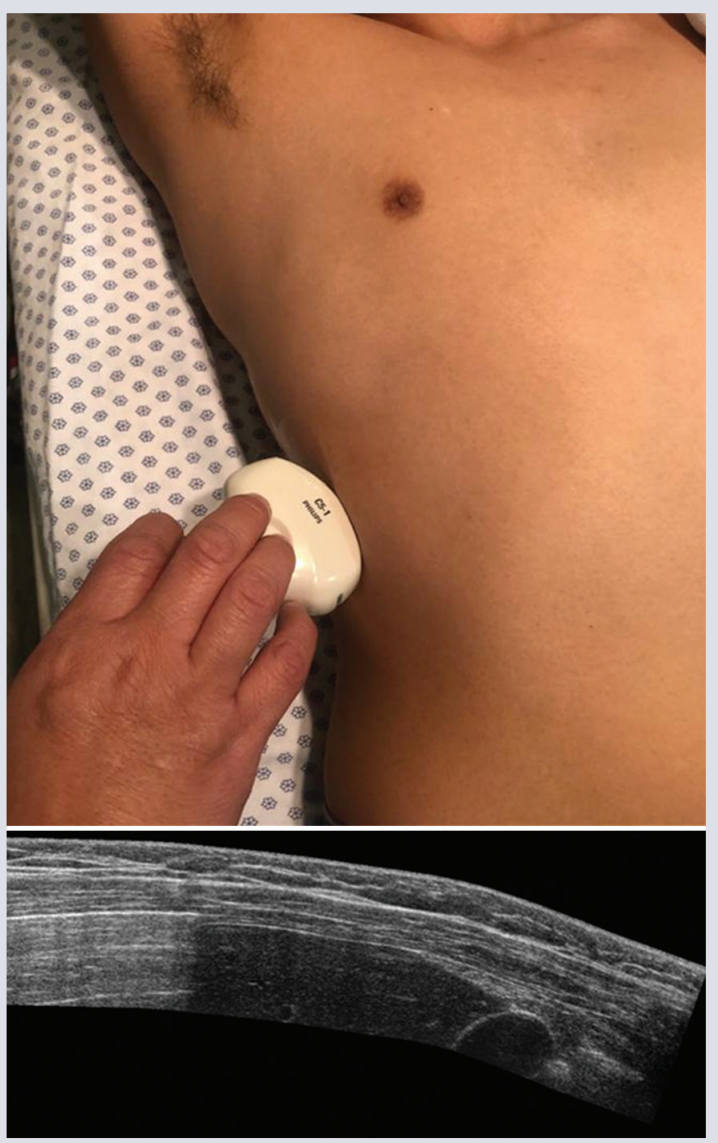

Figura 3. Zona de aposición diafragmática. Se localiza esta zona entre el octavo y el décimo espacios intercostales, entre la línea axilar anterior y medial, en el ángulo costofrénico. El grosor diafragmático debe ser medido a $0.5-2 \mathrm{~cm}$ del ángulo.

La zona de aposición diafragmática representa el 25\% del total de la superficie de la caja torácica durante la respiración, pero puede variar del $0 \%$ a la capacidad pulmonar total a más del 50\% del volumen residual.

Durante la inspiración, la contracción de los músculos costal y crural resulta en un movimiento caudal del diafragma. Este termina en la generación de las fuerzas aposicionales que resultan en un acoplamiento de los músculos abdominales, el diafragma y la caja torácica. Esta fuerza se transmite a través de la zona de aposición y origina la

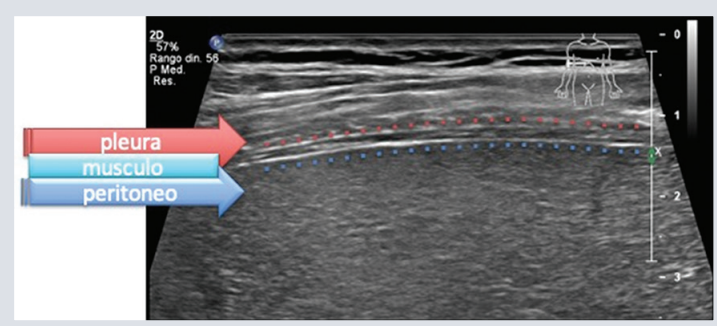

Figura 4. Anatomía ecográfica normal. A nivel de la zona de aposición (abajo) es una estructura de tres capas: pleura, músculo diafragmático y peritoneo. La pleura y el peritoneo se observan lineales e hiperecoicos, y el músculo hipoecoico con imágenes lineales hiperecoicas interpuestas.

expansión de las costillas inferiores. La fuerza aposicional es proporcional a la zona de aposición y la presión abdominal. Simultáneamente, el músculo costal contraído ejerce otra fuerza a los sitios de inserción de las costillas inferiores, que genera un impulsor respiratorio y un movimiento «de asa de cubo o de agarre» ${ }^{5}$.

Para obtener imágenes adecuadas del grosor diafragmático es necesario un transductor de alta frecuencia. A una profundidad de 1.5$3 \mathrm{~cm}$ pueden ser fácilmente identificadas dos líneas paralelas ecogénicas: la línea más cercana es la pleura y la más profunda es el peritoneo. El diafragma es una estructura hipoecoica entre esas dos líneas, con algunas lineales ecogénicas intercaladas por la fascia intermuscular (Fig. 4).

El engrosamiento diafragmático durante una respiración activa refleja la magnitud del esfuerzo respiratorio. El mejor descriptor de este fenómeno es la fracción de contracción diafragmática, que se calcula como la diferencia entre el grosor al final de la inspiración y al final de la espiración (Fig. 5) ${ }^{7}$ : 


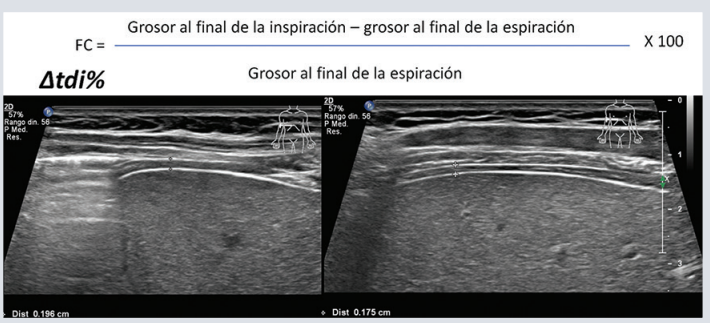

Figura 5. La fracción de contracción se calcula por la diferencia en inspiración y espiración.

Fracción de contracción=

$\frac{\left(\begin{array}{l}\text { Grosor al final de la inspiración } \\ \text { - final de la espiración }\end{array}\right)}{\text { Grosor al final de la espiración }}= \pm 100$

Grosor al final de la espiración

Harper et al. ${ }^{8}$ describen un promedio de fracción de contracción del 20\% durante la respiración corriente, sin diferencias significativas entre el hemidiafragma derecho y el hemidiafragma izquierdo en pacientes ventilados mecánicamente. Otros autores han reportado que las medidas del hemidiafragma izquierdo pueden no ser consistentemente obtenidas como en el derecho, derivado de la aposición de asas de intestino, empobreciendo la venta acústica y, en nuestra experiencia, por una localización más compleja de la zona de aposición izquierda.

\section{Debilidad y parálisis diafragmática}

La parálisis diafragmática y la debilidad pueden ser unilaterales o bilaterales, temporales o permanentes, dependiendo de la causa.

El diagnóstico diferencial de una elevación diafragmática en la radiografía de tórax incluye una placa pobremente inspirada, obesidad con disminución en la distensibilidad, derrame pleural subpulmonar y adherencias pleurales, procesos subdiafragmáticos como ascitis, íleo y organomegalia, entre otros.

En la parálisis unilateral, la sensibilidad de la radiografía de tórax es tan alta como del $90 \%$, mientras que la especificidad es baja $(44 \%)^{9}$.

En la debilidad de uno o ambos hemidiafragmas, la excursión es reducida (menor del 20\% de la fracción de contracción) o tardía en inspiración espontánea o profunda ${ }^{10}$. Además, puede haber limitación de la excursión, y se puede asociar un aumento en el grosor; al contrario de los diafragmas paralizados crónicamente, en los que el grosor suele ser menor de $2 \mathrm{~mm}^{4}$.

Es importante realizar mediciones repetidas, ya que las mediciones únicas pueden originar falsos negativos en situaciones de parálisis aguda en que aún no se ha producido la atrofia, o resultados falsos positivos en individuos pequeños ${ }^{4}$.

Los pacientes con parálisis diafragmática (Fig. 6A) bilateral también pueden tener una evaluación normal. Esto se atribuye a la contracción activa de los músculos abdominales durante la espiración, seguida por una relajación abrupta de los músculos abdominales al comienzo de la inspiración, resultando en un movimiento descendente del diafragma paralizado. Potencialmente, esto puede ser mal interpretado como una contracción diafragmática activa ${ }^{4}$. Esto, seguido de la ausencia de aumento en el grosor con la inspiración, es sugestivo de parálisis diafragmática.

La técnica del olfateo se realiza con esfuerzos inspiratorios cortos solo a través de las 


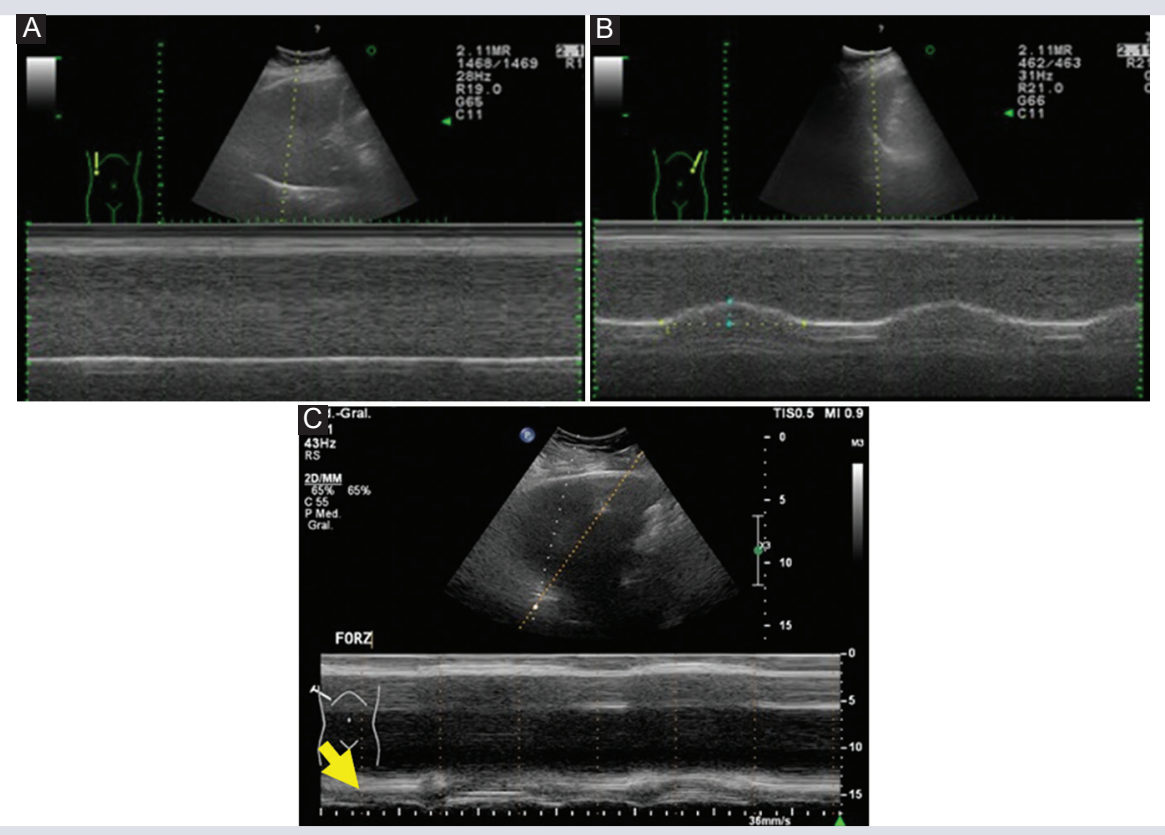

Figura 6. A: parálisis diafragmática. Obsérvese la ausencia de movimiento respiratorio del hemidiafragma derecho. B: el movimiento paradójico se asocia al uso de músculos accesorios y se observa como una inversión del ciclo respiratorio (flecha amarilla).

narinas. En un diafragma paralizado no se documenta movimiento o se mueve en dirección cefálica en vez de hacerlo hacia el transductor. Este movimiento cefálico es exagerado por una técnica de olfateo. El movimiento paradójico del domo es criterio de parálisis diafragmática unilateral. La técnica del olfateo es positiva en más del $90 \%$ de los pacientes con parálisis unilateral (Fig. 6B). Sin embargo, la parálisis diafragmática bilateral no puede ser confirmada por la evaluación del movimiento del domo diafragmático.

Con parálisis bilateral, el diafragma se mueve caudalmente durante la inspiración. Este movimiento caudal está relacionado con dos estrategias compensatorias que los individuos usan para respirar: los músculos intercostales externos para inhalar activamente o los músculos abdominales para inhalar pasivamente. El diafragma puede moverse caudalmente debido a que la presión subdiafragmática se hace más negativa.

Si se confirma el diagnóstico de parálisis diafragmática bilateral, debe tomarse en consideración una evaluación de hipoventilación nocturna ${ }^{1}$.

\section{Disfunción diafragmática inducida por el ventilador}

Al responder a las necesidades del médico clínico, consideramos que es de vital importancia conocer aquellas estrategias por imagen que le permitan modificar, en forma oportuna y rápida, el tratamiento y el pronóstico del paciente. Previa consideración, debemos conocer la disfunción diafragmática inducida por el ventilador, así como su diagnóstico por imagen. 
Un $13 \%$ a $26 \%$ de los pacientes que son extubados tras un ensayo de respiración espontánea necesitan ser reintubados dentro de las siguientes 48 horas $^{7}$.

La ventilación mecánica contribuye a la aparición de miopatía diafragmática y disfunción debido al desuso del diafragma. La disfunción diafragmática inducida por el ventilador se define como una reducción en la capacidad forzada del diafragma y se asocia con un incremento significativo de la mortalidad, así como del tiempo de uso de la ventilación mecánica, haciendo importante identificarla y manejarla en forma temprana.

La disfunción diafragmática está asociada a atrofia grasa y pérdida de las miofibrillas, y puede manifestarse después de 24 a 36 horas de iniciada la ventilación mecánica. Los mecanismos que contribuyen a la disfunción diafragmática inducida por el ventilador incluyen la activación de vías proteolíticas, caspasas y proteosomas, así como la alteración del transporte mitocondrial, resultando en un incremento de las especies reactivas de oxígeno y del estrés oxidativo' ${ }^{1}$.

La evaluación de la función diafragmática mediante fluoroscopia, estimulación del nervio frénico, imagen de resonancia magnética y medición de la presión transdiafragmática tiene ciertas limitaciones, como exposición a radiación ionizante, baja disponibilidad, invasividad y necesidad de trasladar al paciente ${ }^{10}$.

Existen dos predictores ecográficos diafragmáticos para extubar con éxito a un paciente: la excursión diafragmática, que mide la distancia del diafragma durante el ciclo respiratorio, y la fracción de contracción diafragmática, que refleja la variación en el grosor durante el esfuerzo respiratorio, como previamente se ha mencionado.

La definición de «falla al destete» (falla a la extubación) no es estándar, pero cubre uno o más de los siguientes puntos en las primeras 48-72 horas: necesidad de reintubación, ventilación mecánica no invasiva no programada, muerte, traqueostomía, extubación terminal, retardo en la extubación o falla de la prueba de respiración espontánea?

La fracción de contracción ha mostrado ser el mejor estimador del esfuerzo muscular durante la ventilación mecánica no invasiva, y predice la falla a la extubación durante un test de respiración espontánea con el ventilador. El valor de corte reportado de extubación o falla está en el rango del $30 \%$ al $36 \%$ durante el ensayo de respiración espontánea ${ }^{11}$.

El valor de corte para el diagnóstico de disfunción diafragmática usando el modo $\mathrm{M}$, a través de su excursión, es de 10-14 mm durante una prueba de ventilación espontánea con ventilación mecánica ${ }^{11}$.

\section{CONCLUSIONES}

Los hallazgos ecográficos clave para el diagnóstico de enfermedad diafragmática son morfológicos y funcionales.

Las limitantes en la evaluación son:

- Pobre ventana acústica.

- Obesidad.

- Falsos negativos en parálisis diafragmática aguda y falsos positivos en individuos pequeños. 
Se requiere experiencia en la medición de las pautas funcionales del diafragma para poder apoyar al médico clínico con certeza. Cuando se mide la excursión diafragmática, el médico radiólogo debe colocar la línea de medición tan perpendicular como sea posible al diafragma, por lo que la certeza y la repetitividad de la excursión pueden afectarse.

Debe tomarse en consideración que si el punto es la medida de la excursión, y el paciente se encuentra en modo asistido de ventilación mecánica, la excursión puede representar la suma de dos fuerzas ejercidas en la misma dirección: primero, la fuerza de la contracción por sí misma, y segundo, el desplazamiento del diafragma por la presión aplicada del ventilador. En este caso, no hay forma de distinguir qué parte del desplazamiento diafragmático es pasivo, debido a la fuerza externa aplicada por el ventilador, o activo por la acción de contracción conforme se genera una presión negativa. Si el objetivo es evaluar la excursión diafragmática sin la asistencia del ventilador es necesario un breve registro durante la respiración espontánea. De lo contrario, se requiere detectar la contracción y el movimiento diafragmático durante la interacción paciente-ventilador.

\section{FINANCIAMIENTO}

Los autores declaran que no existió financiamiento.

\section{CONFLICTO DE INTERESES}

Ninguno de los autores presenta conflictos de intereses.

\section{RESPONSABILIDADES ÉTICAS}

Protección de personas y animales. Los autores declaran que para esta investigación no se han realizado experimentos en seres humanos ni en animales.

Confidencialidad de los datos. Los autores declaran que han seguido los protocolos de su centro de trabajo sobre la publicación de datos de pacientes.

\section{Derecho a la privacidad y consentimiento} informado. Los autores han obtenido el consentimiento informado de los pacientes y/o sujetos referidos en el artículo. Este documento obra en poder del autor de correspondencia.

\section{BIBLIOGRAFÍA}

1. McCool FD, Manzoor K, Minami T. Disorders of the diaphragm. Clin Chest Med. 2018;39:345-60.

2. Abbey-Mensah GN, Waite S, Reede D, Hassani C, Legasto A. Diaphragm appearance: a clue to the diagnosis of pulmonary and extrapulmonary pathology. Curr Probl Diagn Radiol. 2017;46:47-62.

3. Reiser MF, Adam A, Avni F. Comparative Interpretation of CT and Standard Radiography of the Chest. Coche EE, Ghaye B, de Mey J, Duyck P (Eds.) Springer Berlin Heidelberg, 1a. Edición, Berlin, Alemania, 2011, pp. 229-320.

4. Matamis D, Soilemezi E, Tsagourias M, Akoumianaki E, Dimassi S, Boroli F, et al. Sonographic evaluation of the diaphragm in critically ill patients. Technique and clinical applications. Intensive Care Med. 2013;39:801-10.

5. Haji K, Royse A, Green C, Botha J, Canty D, Royse C. Interpreting diaphragmatic movement with bedside imaging, review article. J Crit Care. 2016;34:56-65.

6. Zanforlin A, Smargiassi A, Inchingolo R, di Marco Berardino A, Valente S, Ramazzina E. Ultrasound analysis of diaphragm kinetics and the diagnosis of airway obstruction: the role of the m-mode index of obstruction. Ultrasound Med Biol. 2014;40:1065-71.

7. Llamas-Álvarez AM, Tenza-Lozano EM, Latour-Pérez J. Diaphragm and lung ultrasound to predict weaning outcome. Chest. 2017;152:1140-50.

8. Harper CJ, Shahgholi L, Cieslak K, Hellyer NJ, Strommen JA, Boon AJ. Variability in Diaphragm Motion During Normal Breathing, Assessed With B-Mode Ultrasound. J Orthop Sports Phys Ther. 2013;43(12):927-31.

9. Kharma N. Dysfunction of the diaphragm: imaging as a diagnostic tool. Curr Opin Pulm Med. 2013;19:394-8.

10. Nason LK, Walker CM, Mcneeley MF, Burivong W, Fligner CL, Godwin JD. Imaging of the diaphragm: anatomy and function. Radiographics. 2012;32:51-71.

11. Zambon M, Greco M, Bocchino S, Cabrini L, Beccaria PF, Zangrillo A Assessment of diaphragmatic dysfunction in the critically ill patient with ultrasound: a systematic review. Intensive Care Med. 2017;43:29-38. 